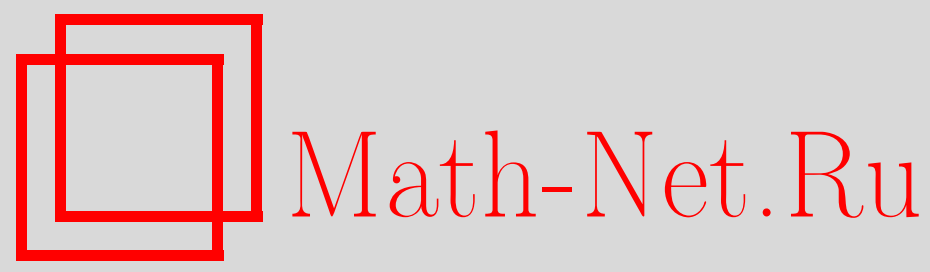

С. Н. Сергеев, Алгоритмическая сложность одной задачи идемпотентно выпуклой геометрии, Матем. заметки, 2003, том 74, выпуск 6, 896-901

DOI: https://doi.org/10.4213/mzm316

Использование Общероссийского математического портала Math-Net.Ru подразумевает, что вы прочитали и согласны с пользовательским соглашением http://www . mathnet.ru/rus/agreement

Параметры загрузки:

IP: 35.173 .219 .12

26 апреля 2023 г., 03:24:45

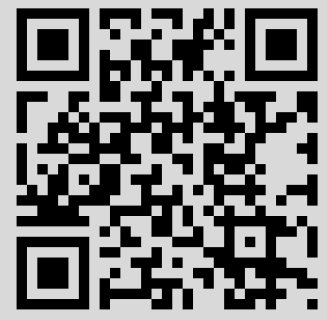




\title{
АЛГОРИТМИЧЕСКАЯ СЛОЖНОСТЬ ОДНОЙ ЗАДАЧИ ИДЕМПОТЕНТНО ВЫПУКЛОЙ ГЕОМЕТРИИ
}

\author{
С. Н. Сергеев
}

\begin{abstract}
В свободном полумодуле над идемпотентным полукольцом $R_{\max } \min$ и в свободном полумодуле над линейно упорядоченным идемпотентным полуполем исследуются свойства идемпотентно выпуклой оболочки двухточечного множества и предлагаются алгоритмы для ее построения.
\end{abstract}

Библиография: 8 названий.

Некоторые асимптотические задачи физики (например, квазиклассическое приближение в квантовой механике [1]), а также многие задачи теории оптимизации, математической экономики и т.п. удается естественно и просто сформулировать в терминах алгебраических структур, включающих операцию максимизации или минимизации [2], [3]. Такие алгебраические структуры являются объектом активно развивающейся идемпотентной математики [3]-[5]. Для интересных, важных и полезных конструкций и результатов традиционной математики над числовьми полями и над аналогичными конструкциями сушествует соответствие с результатами над идемпотентными полуполями и полукольцами, формулируемое в духе принципа соответствия Бора в квантовой теории [6], [7]. Это соответствие может быть, однако, далеким от очевидного. В данной статье рассматривается простейшая задача идемпотентно вьпуклой геометрии (развиваемой, в частности, в [8] и [7]) - построение вьпуклой оболочки двухточечного множества в идемпотентном полумодуле - и устанавливается, что алгоритмическая сложность ее решения растет с повышением размерности полумодуля.

Рассмотрим числовую прямую и введем на ней операции $\oplus=\max , \odot=+$ и добавим элемент $-\infty$, который играет роль $\mathbf{0}$, т.е. наделяется свойствами $-\infty \oplus a=a$, $-\infty \odot a=-\infty$. Операция $\oplus$ удовлетворяет свойствам коммутативности, ассоциативности и идемпотентности $(a \oplus a=a)$, операция $\odot$ является коммутативной, а также дистрибутивной относительно $\oplus$. Указанные свойства рассматриваются в идемпотентном анализе в качестве аксиом идемпотентного полукольиа. Построенная структура обладает еще свойством обратимости операции $\odot$, поэтому называется идемпотентным полуполем $R_{\max }+$.

Идемпотентное полукольцо $R_{\max } \min$ является важным примером полукольца, не являющегося полуполем. Оно включает всю числовую прямую с добавленньми к ней элементами $-\infty$ и $+\infty$ и обладает двумя операциями $\oplus=\max$ и $\odot=\min$. Элементы

Работа выполнена при финансовой поддержке Российского фонда фундаментальных исследований, проект № 02-01-01062. 
$-\infty$ и $+\infty$ объявляются соответственно 0 и 1 полукольца, т.е. наделяются свойствами $-\infty \oplus a=a, a \odot(+\infty)=a$.

Во всех идемпотентных полукольцах операция $\oplus$ индуцирует частичный порядок: $a \preceq b$ тогда и только тогда, когда $a \oplus b=b ; a \prec b$ тогда и только тогда, когда $a \preceq b$ и $a \neq b$. В обоих рассмотренных полукольцах этот порядок линейный, поскольку для любых элементов $a, b$ вьполняется $a \preceq b$ или $b \preceq a$, поэтому для любых элементов $a$ и $b$ этих полуколец определена также операция взятия точной нижней грани $a \wedge b$.

В статье рассматриваются полумодули $S^{n}$ "вектор-столбцов" вида $\left(a^{1}, \ldots, a^{n}\right), a^{i} \in$ $S$, с покоординатными операциями обобщенного сложения и умножения на скаляр из полукольца $S$. Это идемпотентные аналоги векторных пространств. В этих полумодулях операция $\oplus$ индуцирует частичный порядок.

На такой полумодуль легко перенести понятие выпуклости. Множество $C \subset S^{n}$ называется идемпотентно выпуклым, если для любых $x, y \in C$ и $\lambda, \mu \in S$ таких, что $\lambda \oplus \mu=\mathbf{1}$, комбинация $\lambda x \oplus \mu y$ также принадлежит $C$. Точка $y$ назьвается $и д е м-$ потентно выпуклой комбиначией точек $x_{1}, \ldots, x_{m}$, если $y=\bigoplus_{i=1}^{m} \lambda_{i} x_{i}$, причем $\bigoplus_{i=1}^{m} \lambda_{i}=\mathbf{1}$. Здесь и в дальнейшем мы опускаем значок $\odot$ при обозначении обобщенного умножения "вектора" из $S^{n}$ на скаляр из $S$. Идемпотентно выпуклой оболочкой точек $x_{1}, \ldots x_{m} \in S^{n}$ назьвается множество их идемпотентно вьпуклых комбинаций.

В дальнейшем, где это не вызовет недоразумений, мы будем назьвать идемпотентно выпуклые множества, комбинации и оболочки просто вьпуклыми. Все дальнейшие результаты формулируются для полумодулей $S^{n}$, где $S$ - линейно упорядоченное идемпотентное полуполе или полукольцо $R_{\max } \min$.

В полумодулях над этими полукольцами выпуклые комбинации точек $x_{1}$ и $x_{2}$ можно линейно упорядочить следующим образом: $y \preceq x_{1}, x_{2} z$, если $z$ принадлежит вьпуклой оболочке $\left\{y, x_{2}\right\}$. Нетрудно убедиться, что это отношение удовлетворяет всем аксиомам отношения порядка. Теперь естественно дать следующее определение: последовательность выпуклых комбинаций $y_{1}, \ldots, y_{m}$ точек $x_{1}$ и $x_{2}$ назовем монотонной, если $y_{i} \preceq_{x_{1}, x_{2}} y_{i+1}$ для $i=1, \ldots, m-1$. Доказательство следующей леммы очевидно.

Лемма 1. Пусть $y_{1}, \ldots, y_{m} \in S^{n}$ - монотонная последовательность выпуклых комбинаций точек $y_{0}$ и $y_{m+1}$ и $z$ - любая другая выпуклая комбинация $y_{0}$ и $y_{m+1}$. Тогда при некотором единственном $i \in\{0, \ldots, m\}$ точка $z$ является выпуклой комбиначией точек $y_{i}$ и $y_{i+1}$.

Введем еще одно понятие. Пусть $S$ - идемпотентное полуполе или полукольцо $R_{\text {max min. }}$ Множество попарно различных точек $y_{1}, \ldots, y_{m+1} \in S^{n}$ будем назьвать последовательностью вершин выпуклой оболочки точек $y_{1}$ и $y_{m+1}$, если выполнены следуюшие три требования:

1) все $y_{i}$ принадлежат вьпуклой оболочке $y_{1}$ и $y_{m+1}$;

2) для любого $i \in\{1, \ldots, m\}$ точки $y_{i}$ и $y_{i+1}$ принадлежат прямой, задаваемой уравнениями $y^{j}=c^{j}=$ const при $j \in I_{i}$ и $y^{j}=\lambda \odot y_{i}^{j}$ при $j \notin I_{i}$, если $S-$ идемпотентное полуполе, и уравнениями $y^{j}=c^{j}=$ const при $j \in I_{i}$ и $y^{j}=\lambda$ при $j \notin I_{i}$, если $S$ - полукольцо $R_{\max } \min ;$

3) для любого $i \in\{1, \ldots, m-1\}$ множества $I_{i}$ и $I_{i+1}$ не совпадают.

Лемма 2. Последовательность вериин $y_{1}, \ldots, y_{m+1} \in S^{n}$ является монотонной и единственной. 
ДокАЗАТЕЛЬСтво. Если $m=1,2$, то утверждение тривиально. Пусть теперь $m>2$. Докажем по индукции, что последовательность вершин является монотонной. Базис индукции тоже тривиален: $y_{1} \preceq y_{1}, y_{m+1} y_{2}$. Пусть теперь $y_{1} \preceq y_{1}, y_{m+1} \cdots \preceq y_{1}, y_{m+1}$ $y_{k}$. Докажем, что $y_{k} \preceq y_{1}, y_{m+1} y_{k+1}$. Тогда вершина $y_{k+1}$ принадлежит по лемме 1 либо $\left\{y_{k-1}, y_{k}\right\}$, но тогда не вьполнено третье требование определения, либо $\left\{y_{i-1}, y_{i}\right\}$, где $i<k$, но тогда не выполнено второе требование определения. Таким образом, $y_{k} \preceq y_{1}, y_{m+1} y_{k+1}$, и последовательность вершин монотонна по индукции.

Докажем единственность последовательности несовпадающих вершин. Предположим противное. Пусть сушествует другая последовательность вершин $z_{1}, \ldots, z_{s}$. Тогда эта последовательность точек не может быть подпоследовательностью $y_{1}, \ldots, y_{m}$, потому что в этом случае для $z_{l}=y_{i}$ и $z_{l+1}=y_{i+k}$, где $k>1$, не вьполняется второе требование определения. Остается предположить, что, например, точка $z_{j}$ не совпадает ни с одной из вершин $y$. Тогда эта точка принадлежит по лемме 1 одной из выпуклых оболочек $\left\{y_{i}, y_{i+1}\right\}$. Точки $z_{j-1}$ и $z_{j+1}$ должны принадлежать той же выпуклой оболочке, иначе для них не вьполняется второе требование определения. Но в этом случае для точек $z_{j-1}, z_{j}$ и $z_{j+1}$ не вьполняется третье требование определения. Значит, последовательность $z_{1}, \ldots, z_{s}$ совпадает с $y_{1}, \ldots, y_{k}$.

Построим последовательность вершин выпуклой оболочки точек $x_{1}$ и $x_{2}$. Будем рассматривать два случая: когда $x_{1} \prec x_{2}$ и когда $x_{1}$ и $x_{2}$ не сравнимы. Заметим, что без потери обшности можно считать, что $x_{1}^{i} \neq x_{2}^{i}$ для всех $i$.

Докажем следующую теорему для случая $x_{1} \prec x_{2}$.

Теорема 1. Пусть $x_{1}, x_{2} \in S^{n} u x_{1}^{i} \prec x_{2}^{i}$ для всех $i$. Тогда последовательность вериин $y_{i}$ выпуклой оболочки $x_{1}$ и $x_{2}$ существует и с точностью до совпадающих вериин выполнены следующие утверждения:

1) если $S$ - линейно упорядоченное идемпотентное полуполе, то $i=1, \ldots, n+1$, $y_{n+1}=x_{2}$ и $y_{i}=x_{1} \oplus \lambda_{i} x_{2}$, если $i \neq n+1$, әде $\lambda_{i}$ является $i$-м членом последовательности

$$
x_{1}^{l_{1}} \odot\left(x_{2}^{l_{1}}\right)^{-1} \preceq \cdots \preceq x_{1}^{l_{n}} \odot\left(x_{2}^{l_{n}}\right)^{-1},
$$

$u\left(l_{1}, \ldots, l_{n}\right)$ есть соответствующая перестановка $\{1, \ldots, n\}$;

2) если $S$ - полукольцо $R_{\max \min }$, то $i=1, \ldots, 2 n$ и $y_{i}=x_{1} \oplus \lambda_{i} x_{2}$, әде $\lambda_{i}$ является $i$-м членом последовательности

$$
x_{1}^{l(1)} \preceq \cdots \preceq x_{u_{i}}^{l(i)} \preceq \cdots \preceq x_{2}^{l(2 n)}
$$

$u i \mapsto\left(u_{i}, l(i)\right)$ есть соответствующая биекция $\{1, \ldots, 2 n\}$ на $\{1,2\} \times\{1, \ldots, n\}$.

ДокАЗАТЕЛЬСтво. Выбрав из каждого класса эквивалентности совпадающих выпуклых комбинаций $y_{j}$ по представителю и расположив их в порядке возрастания параметра $\lambda$, получим последовательность $z_{1}, \ldots, z_{l+1}$, где $z_{1}=x_{1}, z_{l+1}=x_{2}$ и $z_{j}=$ $y_{r(j)}$, где $r(j)$ - соответствующее отображение множества $\{1, \ldots, l+1\}$ в множество $\{1, \ldots, n+1\}$ в случаеполуполя и в множество $\{1, \ldots, 2 n\}$ в случаеполукольца $R_{\max } \min$. Утверждение теоремы теперь сводится к тому, что последовательность $z_{1}, \ldots, z_{l+1}$ является последовательностью вершин вьпуклой оболочки $x_{1}=z_{1}$ и $x_{2}=z_{l+1}$.

Докажем это утверждение в случае полумодуля над идемпотентным полуполем.

Во-первых, так как $x_{1}^{i} \prec x_{2}^{i}$ для любого $i$, то $\lambda_{i} \prec \mathbf{1}$, следовательно, все $y_{i}$ и $z_{i}$ являются вьпуклыми комбинациями точек $x_{1}$ и $x_{2}$. 
Докажем вьполнение второго и третьего требований определения последовательности вершин. Введем множества индексов

$$
\begin{aligned}
N^{+}(\lambda) & =\left\{i \mid x_{1}^{i} \odot\left(x_{2}^{i}\right)^{-1} \succ \lambda\right\} \\
N^{0}(\lambda) & =\left\{i \mid x_{1}^{i} \odot\left(x_{2}^{i}\right)^{-1}=\lambda\right\}, \\
N^{-}(\lambda) & =\left\{i \mid x_{1}^{i} \odot\left(x_{2}^{i}\right)^{-1} \prec \lambda\right\}
\end{aligned}
$$

и обозначим $y(\lambda)=x_{1} \oplus \lambda x_{2}$. Тогда если $i \in N^{+}(\lambda) \cup N^{0}(\lambda)$, то $y^{i}(\lambda)=x_{1}^{i}$, если $i \in N^{-}(\lambda) \cup N^{0}(\lambda)$, то $y^{i}(\lambda)=\lambda \odot x_{1}^{i}$. Отсюда следует выполнение второго требования определения, причем $I_{j}=N^{+}\left(\lambda_{r(j)+1}\right) \cup N^{0}\left(\lambda_{r(j)+1}\right)$ для любых $j=1, \ldots, l$. Заметим, что индекс $l_{r(j)}$ принадлежит $I_{j-1}$, но не принадлежит $I_{j}$, поэтому третье требование также вьполнено.

Докажем утверждение теоремы для полумодуля над полукольцом $R_{\max } \min$.

Поскольку в полукольце $R_{\max }$ min никакие элементы не превосходят $\mathbf{1}$, первое требование определения вершин вьпуклой оболочки выполнено тривиально.

Докажем выполнение второго и третьего требований. Введем множества индексов

$$
\begin{array}{rlrl}
N_{1}^{+}(\lambda) & =\left\{i \mid x_{1}^{i} \succ \lambda\right\}, & & N_{1}^{0}(\lambda)=\left\{i \mid x_{1}^{i}=\lambda\right\}, \\
N_{1}^{-}(\lambda)=\left\{i \mid x_{1}^{i} \prec \lambda\right\}, & & N_{2}^{+}(\lambda)=\left\{i \mid x_{2}^{i} \succ \lambda\right\}, \\
N_{2}^{0}(\lambda)=\left\{i \mid x_{2}^{i}=\lambda\right\}, & & N_{2}^{-}(\lambda)=\left\{i \mid x_{2}^{i} \prec \lambda\right\} .
\end{array}
$$

Тогда если $i \in N_{2}^{-}(\lambda) \cup N_{2}^{0}(\lambda)$, то $y^{i}(\lambda)=x_{2}^{i}$, если $i \in\left(N_{2}^{+}(\lambda) \cup N_{2}^{0}(\lambda)\right) \cap\left(N_{1}^{+}(\lambda) \cup\right.$ $\left.N_{1}^{0}(\lambda)\right)$, то $y^{i}(\lambda)=x_{1}^{j}$, если $j \in\left(N_{2}^{+}(\lambda) \cup N_{2}^{0}(\lambda)\right) \cap\left(N_{1}^{-}(\lambda) \cup N_{1}^{0}(\lambda)\right)$, то $y^{i}(\lambda)=\lambda$. Отсюда следует выполнение второго требования, причем $I_{j}=\left(N_{2}^{-}\left(\lambda_{r(j)}\right) \cup N_{2}^{0}\left(\lambda_{r(j)}\right)\right) \cup$ $\left(\left(N_{2}^{+}\left(\lambda_{r(j)+1}\right) \cup N_{2}^{0}\left(\lambda_{r(j)+1}\right)\right) \cap\left(N_{1}^{+}\left(\lambda_{r(j)+1}\right) \cup N_{1}^{0}\left(\lambda_{r(j)+1}\right)\right)\right)$ для любых $j=1, \ldots, l$. Индекс $l(r(j))$ не может принадлежать одновременно $I(j-1)$ и $I(j)$, поэтому третье требование также выполнено.

Лемма 2 гарантирует единственность последовательности $z_{1}, \ldots, z_{l+1}$.

Докажем теперь аналогичную теорему для второго случая. Введем обозначения

$$
M_{0}=\left\{i \mid x_{1}^{i}=x_{2}^{i}\right\}, \quad M_{1}=\left\{i \mid x_{2}^{i} \prec x_{1}^{i}\right\}, \quad M_{2}=\left\{i \mid x_{1}^{i} \prec x_{2}^{i}\right\} .
$$

Теорема 2. Пусть точки $x_{1}, x_{2} \in S^{n}$ не сравнимы, $M_{0}=\varnothing$, тогда последовательность вериин их выпуклой оболочки существует и единственна.

ДокАЗАТЕльство. Для случая $x_{1} \prec x_{2}$ предложение уже доказано. Осталось доказать его для случая, когда $x_{1}$ и $x_{2}$ не сравнимы. В этом случае $\{1, \ldots, n\}=M_{1} \cup M_{2}$. Заметим, что точка $x_{1} \oplus x_{2}$ является вьпуклой комбинацией точек $x_{1}$ и $x_{2}$ и что в данном случае она отлична от них. Согласно лемме 1 все выпуклые комбинации точек $x_{1}$ и $x_{2}$ принадлежат либо вьпуклой оболочке $\left\{x_{1}, x_{1} \oplus x_{2}\right\}$, либо вьпуклой оболочке $\left\{x_{1} \oplus x_{2}, x_{2}\right\}$. Так как $x_{1} \prec x_{1} \oplus x_{2}$ и $x_{2} \prec x_{1} \oplus x_{2}$, то для обеих выпуклых оболочек существует последовательность промежуточных вершин. Соединяя две последовательности вершин с помошью точки $x_{1} \oplus x_{2}$, мы получаем последовательность вершин выпуклой оболочки $x_{1}$ и $x_{2}$. Для всех вершин, кроме $x_{1} \oplus x_{2}$ все требования определения автоматически выполняются, как и первые два требования для точки $x_{1} \oplus x_{2}$. Докажем 
выполнение третьего требования. Действительно, вьпуклая оболочка $\left\{x_{1}, x_{1} \oplus x_{2}\right\}$ принадлежит плоскости $x^{i}=x_{1}^{i}, i \in M_{1}$, а выпуклая оболочка $\left\{x_{1} \oplus x_{2}, x_{2}\right\}$ - плоскости $x^{i}=x_{2}^{i}, i \in M_{2}$, причем $M_{1} \cap M_{2}=0$. Это означает, что множества $I$ для точки $x_{1} \oplus x_{2}$ и соседних с ней вершин не совпадают.

Единственность гарантируется леммой 2.

На основании теорем 1 и 2 предложим следуюшие алгоритмы построения последовательности несовпадающих вершин вьпуклой оболочки точек $x_{1}$ и $x_{2}$ в полумодуле $S^{n}$ над идемпотентным полуполем и над полукольцом $R_{\max } \min$.

Вначале для обоих алгоритмов исходное множество индексов $\{1, \ldots, n\}$ разбивается на подмножества $M_{1}, M_{2}$ и $M_{0}$. Далее, если $S$ - полуполе, для индексов $i$, принадлежаших $M_{1}$, производится сортировка произведений $x_{1}^{i} \odot\left(x_{2}^{i}\right)^{-1}$ по возрастанию, в результате получается последовательность $\lambda_{1} \preceq \cdots \preceq \lambda_{m}$, где $\lambda_{i}=x_{1}^{l_{i}} \odot\left(x_{2}^{l_{i}}\right)^{-1}$. Для $i$, принадлежащих $M_{2}$, производится сортировка $x_{2}^{i} \odot\left(x_{1}^{i}\right)^{-1}$ и получается последовательность $\mu_{1} \preceq \cdots \preceq \mu_{k}$, где $\mu_{i}=x_{2}^{m_{i}} \odot\left(x_{1}^{l_{i}}\right)^{-1}$. Если $S$ - полукольцо $R_{\max \min }$, для индексов $i$, принадлежащих $M_{1}$, производится совместная сортировка $x_{1}^{i}$ и $x_{2}^{i}$ по возрастанию, в результате получается последовательность $\lambda_{1} \preceq \cdots \preceq \lambda_{2 m}$, где $\lambda_{i}=x_{u_{i}}^{l(i)}$. Для $i$, принадлежащих $M_{2}$, производится аналогичная сортировка и получается последовательность $\mu_{1} \preceq \cdots \preceq \mu_{2 k}$, где $\mu_{i}=x_{s_{i}}^{m(i)}$ Далее строится последовательность вершин вьпуклых оболочек $\left\{x_{1}, x_{1} \oplus x_{2}\right\}$ и $\left\{x_{1} \oplus x_{2}, x_{2}\right\}$. Приведем алгоритмы построения последовательности вершин первой из этих вьпуклых оболочек. Алгоритм построения второй можно получить путем замены $x_{1}$ на $x_{2}, M_{1}$ на $M_{2}$ и (в случае полукольца $\left.R_{\max \min }\right) N_{1}^{+,-, 0}$ на $N_{2}^{+,-, 0}$.

\section{Алгоритм построения последовательности вершин выпуклой оболочки} $x_{1}$ и $x_{1} \oplus x_{2}$ в полумодуле над идемпотентным полуполем.

Состояние вычислительного процесса. Множество $\{1, \ldots, n\}$ разбито на непересекающиеся множества $M_{1}, M_{2}$ и $M_{0}$, первое из которых разбито на непересекающиеся множества $N^{-}, N^{0}$ и $N^{+}$. Исходя из принадлежности к этим множествам соответствуюших индексов, вычисляются координаты вершин.

Начальное состояние: $N^{0}=\left\{l_{1}\right\}, N^{+}=M_{1} \backslash\left\{l_{1}\right\}$.

Стандартный шаг алгоритма. Если $\lambda_{i+1} \succ \lambda_{i}$, то индекс $l_{i+1}$ заносится в $N^{0}$, индекс $l_{i}$ заносится в $N^{-}$и вычисляются координаты вершины $y_{i+1}$ по следующим правилам: $y_{i+1}^{j}=\lambda_{i+1} \odot x_{2}^{j}$, если $j \in\left(N^{-} \cup N^{0}\right)$, и $y_{i+1}^{j}=x_{1}^{j}$, если $j \in N^{+} \cup M_{2} \cup M^{0}$.

Если $\lambda_{i+1}=\lambda_{i}$, то индекс $l_{i+1}$ заносится в $N^{0}$ и больше никаких действий не производится.

Алгоритм построения последовательности вершин выпуклой оболочки $x_{1}$ и $x_{1} \oplus x_{2}$ в полумодуле над полукольцом $R_{\max \min }$.

Состояние вычислительного процесса. Множество $\{1, \ldots, n\}$ разбито на непересекающиеся множества $M_{1}, M_{2}$ и $M_{0}$, первое из которых разбито на непересекающиеся множества $N_{1}^{-}, N_{1}^{0}$ и $N_{1}^{+}$, а также на $N_{2}^{-}, N_{2}^{0}$ и $N_{2}^{+}$. Исходя из принадлежности к этим множествам соответствуюших индексов вычисляются координаты вершин.

Начальное состояние: $N_{1}^{0}=\left\{l_{1}\right\}, N_{1}^{+}=M_{1} \backslash\left\{l_{1}\right\}, N_{2}^{0}=0, N_{2}^{+}=M_{1}$.

Стандартный шаг алгоритма. Если $\lambda_{i+1} \succ \lambda_{i}$, то индекс $l(i+1)$ заносится в $N_{u_{i+1}}^{0}$, индекс $l(i)$ заносится в $N_{u_{i+1}}^{-}$и вычисляются координаты вершины $y_{i+1}$ по следуюшим правилам: $y_{i+1}^{j}=\lambda_{i+1}$, если $j \in\left(N_{1}^{-} \cup N_{1}^{0}\right) \cap\left(N_{2}^{+}\right), y_{i+1}^{j}=x_{2}^{j}$, если $j \in\left(N_{2}^{-} \cup N_{2}^{0}\right)$, и $y_{i+1}^{j}=x_{1}^{j}$, если $j \in\left(N_{2}^{+} \cap N_{1}^{+}\right) \cup M_{2} \cup M_{0}$. 
Если $\lambda_{i+1}=\lambda_{i}$, то индекс $l(i+1)$ заносится в $N_{u_{i+1}}^{0}$ и больше никаких действий не производится.

Построенные алгоритмы имеют вычислительную сложность $n^{2}$. Это объясняется тем, что количество вершин (не считая исходных точек) может достигать $n-1$ для полуполя и $2 n-2$ для $R_{\max } \min$.

В заключение отметим связь последовательности вершин вьпуклой оболочки и теории (идемпотентно) линейньх функционалов в идемпотентных полумодулях, развитой в статье [7]. В этой статье $x$-функиионалом называется функционал, определяемый формулой

$$
y \longmapsto x^{*}(y)=\inf \{k \in S \mid k x \succeq y\},
$$

где $S$ - идемпотентное полукольцо, $x$ и $y$ - элементы полумодуля над этим полукольцом. Доказьвается, что если $S$ является идемпотентным полуполем и полумодуль удовлетворяет некоторым естественным требованиям, то $x$-функционал является линейным и любой линейный функционал представляется в виде $x$-функционала. Заметим теперь, что в полумодуле $S^{n}$, где $S$ - идемпотентное полуполе, $x^{*}(y)=\bigoplus_{i}\left(x^{i}\right)^{-1} \odot y^{i}$. Тогда, если $x_{1}^{i} \prec x_{2}^{i}$ для любого $i$, то $y_{n}=x_{1} \oplus x_{2}^{*}\left(x_{1}\right) x_{2}=x_{2}^{*}\left(x_{1}\right) x_{2}$. Одна из координат $y_{n}$ совпадает с соответствуюшей координатой $x_{1}$, значит, все остальные вершины ее автоматически наследуют. Рассматривая $x_{1}$ и $y_{n}$ как точки на $(n-1)$-мерной плоскости, найдем, что $y_{n-1}=y_{n}^{*}\left(x_{1}\right) y_{n}$. Аналогично могут быть найдены все оставшиеся вершины.

Автор благодарит А. Н. Соболевского за постоянное внимание к работе и ценные указания.

\section{СПИСОК ЦИТИРОВАННОЙ ЛИТЕРАТУРЫ}

[1] Маслов В. П. Асимптотические методы решения псевдодифференциальных уравнений. М.: Наука, 1987.

[2] Bacelli F. L., Cohen G., Olsder G. J., Quadrat J. P. Synchronization and Linearity: an Algebra for Discrete Event Systems. New York et al.: John Wiley \& Sons Publ., 1992.

[3] Маслов В. П., Колокольцов В. Н. Идемпотентный анализ и его применение в оптимальном управлении. М.: Наука, 1994.

[4] Maslov V. P., Samborski i S. N. (eds.) Idempotent Analysis. Adv. Sov. Math. V. 13. Providence, R.I.: Amer. Math. Soc., 1992.

[5] Gunawardena J. (ed.) Idempotency. Publ. of the Newton Institute. Cambridge: Cambridge Univ. Press, 1998.

[6] Litvinov G. L., Maslov V. P. Correspondence Principle for Idempotent Calculus and Some Computer Applications. Bures-Sur-Yvette: Institut des Hautes Etudes Scientifiques, 1995. (IH$\mathrm{ES} / \mathrm{M} / 95 / 33)$.

[7] Литвинов Г. Л., Маслов В. П., Шпиз Г. Б. Идемпотентньй функциональный анализ. Алгебраический подход // Матем. заметки. 2001. Т. 69. № 5. С. 758-797.

[8] Zimmermann K. A general separation theorem in extremal algebras // Ekonomicko-matematicky obzor (Prague). 1977. V. 13. № 2. P. 179-201. 